PROCEEDINGS OF THE

AMERICAN MATHEMATICAL SOCIETY

Volume 126, Number 1, January 1998, Pages 127-133

S 0002-9939(98)03967-7

\title{
CONTINUOUS MULTIPLICATIVE MAPPINGS ON $C(X)$
}

\author{
GORAZD LEŠNJAK AND PETER ŠEMRL
}

(Communicated by Palle E. T. Jorgensen)

\begin{abstract}
Let $X$ and $Y$ be compact Hausdorff topological spaces, and let $C(X)$ and $C(Y)$ be real Banach algebras of all real-valued continuous functions on $X$ and $Y$, respectively. The general form of continuous multiplicative mappings $\Phi: C(X) \rightarrow C(Y)$ is given.
\end{abstract}

Let $X$ and $Y$ be compact Hausdorff topological spaces, and let $C(X)$ and $C(Y)$ be real Banach algebras of all real-valued continuous functions on $X$ and $Y$, respectively. We denote by $\mathbb{1}_{X}, \mathbb{O}_{X} \in C(X)$ the functions defined by $\mathbb{1}_{X}(x)=1$ and $\mathbb{O}_{X}(x)=0$ for all $x \in X$. It is well known that every unital ring homomorphism (additive and multiplicative mapping sending $\mathbb{1}_{X}$ into $\left.\mathbb{1}_{Y}\right) \Phi: C(X) \rightarrow C(Y)$ is a composition operator, that is, there exists a continuous mapping $\varphi: Y \rightarrow X$ such that $\Phi(f)=f \circ \varphi$ for every $f \in C(X)$. The mapping $\Phi$ is a ring isomorphism if and only if $\varphi$ is a homeomorphism. This means in particular, that the ring $C(X)$ determines the space $X$ up to a homeomorphism. Even more is true, namely, the algebra $C(X)$ is completely determined by its multiplicative structure. More precisely, $C(X)$ and $C(Y)$ are isomorphic as multiplicative semigroups if and only if the underlying spaces $X$ and $Y$ are homeomorphic. This follows from Milgram's representation of the general bijective multiplicative mappings from $C(X)$ onto $C(Y)[3]$. An interested reader can find some related results on multiplicative isomorphisms and multiplicative derivations in [2], [4], [5].

In this note we will study multiplicative mappings from $C(X)$ into $C(Y)$ that are not bijective. It seems hopeless to find a general representation theorem for such mappings without imposing any additional assumptions. We believe that besides the bijectivity the continuity assumption is the most natural one. The special case $\Phi: C(X) \rightarrow \mathbb{R}$ (this means that $Y$ consists of one point only) has been independently treated by Turowicz [6] and Bourgin [1]. If $\Phi: C(X) \rightarrow \mathbb{R}$ is a continuous multiplicative functional, then the same holds true for the mapping $|\Phi|$. So, in order to solve the problem of determining the general form of continuous multiplicative functionals on $C(X)$ one has to consider first the general form of positive nontrivial continuous multiplicative functionals. Here, the positivity is defined as $\Phi(f) \geq 0$ for all $f \in C(X)$ and the nontriviality means that $\Phi$ is neither identically zero nor identically one. Bourgin [1] and Turowicz [6] proved the following result.

Theorem 1. Let $\Phi: C(X) \rightarrow \mathbb{R}$ be a positive nontrivial continuous multiplicative functional. Then there exists a positive regular Borel measure $\mu$ on $X$ with at most

Received by the editors April 30, 1996.

1991 Mathematics Subject Classification. Primary 46E25, 46J10.

This work was supported by the Ministry of Science and Technology of Slovenia.

(C)1998 American Mathematical Society 
countable support $S=\left\{s_{1}, s_{2}, s_{3}, \ldots\right\}$ satisfying $\mu\left(\left\{s_{i}\right\}\right)>0, i=1,2,3, \ldots$, such that

$$
\Phi(f)= \begin{cases}0 & \text { if } f\left(s_{i}\right)=0 \text { for some positive integer } i, \\ e^{\int \log |f| d \mu} & \text { otherwise. }\end{cases}
$$

Now, if $\Phi: C(X) \rightarrow \mathbb{R}$ is an arbitrary continuous multiplicative functional, then we can write $\Phi$ as $\Phi(f)=|\Phi|(f) \sigma(f)$ where $\sigma$ maps $C(X)$ into $\{-1,0,1\}$. Of course, one can define the values of $\sigma$ at functions $f$ satisfying $\Phi(f)=0$ arbitrarily, but throughout this note we will always assume that $\sigma(f)=0$ for such functions. Bourgin [1] observed that the value of $\sigma(f)$ is completely determined by the values of function $f$ on $S$. Turowicz [6] gave an explicit expression of $\sigma(f)$ depending on values $\operatorname{sign}(f)$ on $S$ and two countable families of parameters $\alpha_{i}, \beta_{i}, i=1,2,3, \ldots$. In his formula the $\alpha$ 's are uniquely determined by the functional $\Phi$ while the values of parameters $\beta_{1}, \beta_{2}, \beta_{3}, \ldots$ depend not only on $\Phi$ but also on the ordering of the countable set $S$.

It seems that the most natural approach when studying continuous multiplicative mappings $\Phi: C(X) \rightarrow C(Y)$ is to compose $\Phi$ with $\delta_{y}, y \in Y$, and then to apply the results on multiplicative functionals. Here, $\delta_{y}: C(Y) \rightarrow \mathbb{R}$ is the evaluation at the point $y \in Y$. It turns out that Turowicz's description of the mapping $\sigma$ is not suitable for this approach because of being nonunique. To avoid this obstacle we will give a different description of $\sigma$ and then apply this result to find a general form of continuous multiplicative mappings from $C(X)$ into $C(Y)$.

We start with some definitions. Let $S$ be a closed subset of a compact set $X$, and let $\mathcal{F}$ be any family of open (with respect to the relative topology on $S$ ) and closed subsets of $S$. Then $\mathcal{F}$ is called a sign-determining family on $S$ if

$$
A \triangle B \in \mathcal{F} \Leftrightarrow \text { exactly one of sets } A \text { and } B \text { belongs to } \mathcal{F}
$$

for every pair of open and closed subsets $A, B \subset S$. Here, $A \triangle B$ is defined as $A \triangle B=(A \backslash B) \cup(B \backslash A)$. Note that the empty set does not belong to any signdetermining family. Let $f$ be any real-valued continuous function on $X$. We denote by $N_{S}(f)$ the set of all points $x \in S$ satisfying $f(x)<0$. For a given signdetermining family $\mathcal{F}$ on $S$ we define a mapping $\sigma_{\mathcal{F}}: C(X) \rightarrow \mathbb{R}$ by

$$
\sigma_{\mathcal{F}}(f)=\left\{\begin{array}{rl}
0 & \text { there exists } x \in S \text { such that } f(x)=0, \\
1 & f(x) \neq 0 \text { for all } x \in S \text { and } N_{S}(f) \notin \mathcal{F}, \\
-1 & f(x) \neq 0 \text { for all } x \in S \text { and } N_{S}(f) \in \mathcal{F} .
\end{array}\right.
$$

From $N_{S}(f g)=N_{S}(f) \triangle N_{S}(g)$ it follows that $\sigma_{\mathcal{F}}$ is multiplicative. Note that in the special case that $\mathcal{F}$ is the empty family we get $\sigma_{\mathcal{F}}(f)=1$ for all functions $f$ without zeroes in $S$.

Now we are ready to prove our first result on multiplicative functionals.

Theorem 2. A mapping $\Phi: C(X) \rightarrow \mathbb{R}$ is a nontrivial continuous multiplicative functional if and only if there exists a positive regular Borel measure $\mu$ on $X$ with at most countable support $S=\left\{s_{1}, s_{2}, s_{3}, \ldots\right\}$ satisfying $\mu\left(\left\{s_{i}\right\}\right)>0, i=1,2,3, \ldots$, and a sign-determining family $\mathcal{F}$ on $S$ such that

$$
\Phi(f)= \begin{cases}0 & \text { if } f\left(s_{i}\right)=0 \text { for some positive integer } i, \\ \sigma_{\mathcal{F}}(f) e^{\int \log |f| d \mu} & \text { otherwise. }\end{cases}
$$


Proof. Let us first assume that $\Phi$ is a nontrivial continuous multiplicative functional. According to Theorem 1 we can find a measure $\mu$ with the support $S$ satisfying all the desired properties such that the functional $|\Phi|$ is represented by (1). Let $A$ be any open and closed subset of $S$. Then $\chi_{S \backslash A}-\chi_{A}: S \rightarrow \mathbb{R}$ is a continuous function. So, by Tietze's extension theorem there exists a continuous extension $g_{A}$ of this function over $X$. Note that the restriction of $g_{A}^{2}$ to $S$ is identically equal to one. Consequently, $\left(\Phi\left(g_{A}\right)\right)^{2}=1$, and therefore either $\Phi\left(g_{A}\right)=1$ or $\Phi\left(g_{A}\right)=-1$. We define $\mathcal{F}$ to be the set of all open and closed subsets $A \subset S$ satisfying $\Phi\left(g_{A}\right)=-1$. We have already mentioned that the value of $\Phi(f)$ is completely determined by the values of functions $f$ on $S$. Hence, $\mathcal{F}$ is well defined. A straightforward computation shows that $\mathcal{F}$ is a sign-determining family. In order to see that (3) is satisfied for all functions from $C(X)$ we take a function $f \in C(X)$ having no zeroes in $S$. From $\Phi\left(|f|^{2}\right)=\Phi\left(f^{2}\right)=(\Phi(f))^{2}$ it follows that $\Phi(|f|)=|\Phi(f)|$. It is also clear that $f g_{N_{S}(f)}$ is a strictly positive function on $S$. Hence, it is sent by $\Phi$ into a positive real number, and consequently,

$$
\Phi(f)=\sigma_{\mathcal{F}}(f) e^{\int \log |f| d \mu} .
$$

To prove the converse we assume that a measure $\mu$ with the support $S$ and a signdetermining family $\mathcal{F}$ on $S$ satisfy all the assumptions of our theorem. Obviously, the functional $\Phi$ given by (3) is nontrivial and multiplicative. We have to prove that it is also continuous at every $f \in C(X)$. Let us assume first that $\Phi(f)=0$. It follows that there exists $x \in S$ such that $f(x)=0$. Let $g$ be any function without zeroes in $S$. The continuity of $\Phi$ at $f$ follows from

$$
|\Phi(g)| \leq e^{\mu(\{x\}) \log |g(x)|} e^{\int_{X \backslash\{x\}} \log (\|f\|+\|g-f\|) d \mu} .
$$

The mapping

$$
f \mapsto \log \Phi\left(e^{f}\right)=\int f d \mu, \quad f \in C(X),
$$

is continuous which further yields the continuity of $\Phi$ at every positive function $f$. The value of $\Phi(f)$ depends only on the values of $f$ on the set $S$, and consequently, $\Phi$ is continuous at every function which is positive on $S$. It follows that $|\Phi|$ is continuous. It remains to see that for every $f \in C(X)$ without zeroes in $S$ there exists a positive number $\eta$ such that $\|g-f\|<\eta$ implies $\sigma_{\mathcal{F}}(g)=\sigma_{\mathcal{F}}(f)$. If we take $\eta=\min _{x \in S}\{|f(x)|\}$, then $\|g-f\|<\eta$ yields that $g$ is without zeroes in $S$ and $N_{S}(g)=N_{S}(f)$ as required. This completes the proof.

In order to get a complete description of continuous multiplicative mappings $\Phi: C(X) \rightarrow C(Y)$ we need the following definitions. Let $X$ and $Y$ be compact Hausdorff spaces. Assume that we have associated to each $y \in Y$ a closed subset $S_{y} \subset X$. The mapping $y \mapsto S_{y}$ is continuous at the point $y_{0} \in Y$ if

- for every neighbourhood $U$ of $S_{y_{0}}$ there exists a neighbourhood $V$ of $y_{0}$ such that $S_{z} \subset U$ for every $z \in V$, and

- for every point $x \in S_{y_{0}}$ and every neighbourhood $W$ of $x$ there exists a neighbourhood $T$ of $y_{0}$ such that $S_{z} \cap W \neq \varnothing$ for every $z \in T$.

The mapping $y \rightarrow S_{y}$ is continuous if it is continuous at every point $y_{0} \in Y$.

Assume further that we have associated to each $y \in Y$ also a sign-determining family $\mathcal{F}_{y}$ on $S_{y}$. The mapping $y \mapsto \mathcal{F}_{y}$ is continuous at $y_{0} \in Y$ if for every open 
and closed set $A \subset S_{y_{0}}$ and every pair of open disjoint subsets $U, V \subset X$ satisfying $A \subset U$ and $S_{y_{0}} \backslash A \subset V$ there exists a neighbourhood $W$ of $y_{0}$ such that

$$
A \in \mathcal{F}_{y_{0}} \Leftrightarrow S_{z} \cap U \in \mathcal{F}_{z}
$$

for every $z \in W$. Of course, this definition makes sense only under the assumption that the mapping $y \mapsto S_{y}$ is continuous at $y_{0}$. Namely, in this case we can find a neighbourhood $T$ of $y_{0}$ such that $S_{z} \subset U \cup V$ for every $z \in T$. It follows that $S_{z} \cap U$ is an open and closed subset of $S_{z}$. As before, we say that the mapping $y \mapsto \mathcal{F}_{y}$ is continuous if it is continuous at every point $y_{0} \in Y$.

Finally, let us assume that $\left\{\mu_{y}: y \in Y\right\}$ is a family of positive Borel measures on $X$. We will call the mapping $y \mapsto \mu_{y}$ lower semicontinuous at $y_{0} \in Y$ if for every open $U \subset X$ and every positive real number $\varepsilon$ there exists a neighbourhood $V$ of $y_{0}$ such that $\mu_{z}(U)>\mu_{y_{0}}(U)-\varepsilon$ for every $z \in V$. This mapping will be called upper semicontinuous at $y_{0} \in Y$ if for every compact $K \subset X$ and every positive real number $\varepsilon$ there exists a neighbourhood $V$ of $y_{0}$ such that $\mu_{z}(K)<\mu_{y_{0}}(K)+\varepsilon$ for every $z \in V$. The mapping $y \mapsto \mu_{y}$ is continuous if it is upper and lower semicontinuous at every point $y_{0} \in Y$.

Theorem 3. Let $X$ and $Y$ be compact Hausdorff topological spaces. A mapping $\Phi: C(X) \rightarrow C(Y)$ is a continuous multiplicative mapping if and only if

$$
\Phi(f)(y)= \begin{cases}0 & y \in Y_{0}, \\ 1 & y \in Y_{1}, \\ 0 & y \in Z \text { and } f \text { has a zero in } S_{y}, \\ \sigma_{\mathcal{F}_{y}}(f) e^{\int \log |f| d \mu_{y}} & y \in Z \text { and } f \text { has no zeroes in } S_{y},\end{cases}
$$

where $Y_{0}, Y_{1}$, and $Z$ are disjoint closed subsets of $Y$ such that $Y=Y_{0} \cup Y_{1} \cup Z$, $\mu_{y}$ is a positive measure on $X$ having all the properties as in Theorem 1 for every $y \in Z, \mathcal{F}_{y}$ is a sign-determining family on the support $S_{y}$ of $\mu_{y}$ for every $y \in Z$, and the mappings $y \mapsto \mu_{y}, y \mapsto S_{y}, y \rightarrow \mathcal{F}_{y}$ are continuous on $Z$.

Proof. Let us first assume that $\Phi$ is a continuous multiplicative mapping. Clearly, the functions $\Phi\left(\mathbb{1}_{X}\right), \Phi\left(\mathbb{O}_{X}\right) \in C(Y)$ are idempotents. It follows that $Y_{0}=$ $\Phi\left(\mathbb{1}_{X}\right)^{-1}(\{0\})$ and $Y_{1}=\Phi\left(\mathbb{O}_{X}\right)^{-1}(\{1\})$ are open and closed subsets of $Y$. Applying the multiplicativity of $\Phi$ it is easy to see that the functional $\delta_{y} \circ \Phi$ is identically equal to zero for every $y \in Y_{0}$ and similarly, $\delta_{y} \circ \Phi$ must be identically equal to one for every $y \in Y_{1}$. In particular, $Y_{0}$ and $Y_{1}$ are disjoint subsets of $Y$. Set $Z=Y \backslash\left(Y_{0} \cup Y_{1}\right)$. Obviously, the multiplicative functional $\delta_{y} \circ \Phi$ is nontrivial for every $y \in Z$. According to Theorem 2 we can associate to each $y \in Z$ a positive regular Borel measure $\mu_{y}$ on $X$ with a countable support $S_{y}$ and a sign-determining family $\mathcal{F}_{y}$ on $S_{y}$ such that $\delta_{y} \circ \Phi$ has a representation of the form (3). We have to show now that the mappings $y \mapsto \mu_{y}, y \mapsto S_{y}$, and $y \mapsto \mathcal{F}_{y}$ are continuous.

Let us choose $y \in Z$ and a neighbourhood $U$ of $S_{y}$. By Urysohn's lemma there exists a continuous function $f \in C(X)$ with supp $f \subset U$ such that its restriction to $S_{y}$ is identically equal to one. It is easy to see that $\Phi(f)(y)=1$. As $\Phi(f)$ is continuous at $y$ we can find a neighbourhood $V \subset Z$ of $y$ such that $\Phi(f)(z)>1 / 2$ for every $z \in V$. This yields that $f$ has no zeroes in $S_{z}$ for every $z \in V$ which further implies that $S_{z} \subset U$ for every $z \in V$. Let us now assume that $x \in S_{y}$, and that $W$ is a neighbourhood of $x$. Introducing a function $g \in C(X)$ with supp $g \subset\{x\}^{C}$ satisfying $g(u)=1$ for all $u \in W^{C}$ one can prove similarly as above that $S_{z} \cap W \neq \varnothing$ 
for every $z \in\{w \in Z: \Phi(g)(w)<1 / 2\}$. Hence, the mapping $y \mapsto S_{y}$ is continuous on $Z$.

In the next step we will prove that $y \mapsto \mu_{y}$ is a continuous mapping on $Z$. Let $K$ be any compact subset of $X, \varepsilon$ a positive real number, and let $y$ be any point in $Z$. The regularity of $\mu_{y}$ implies the existence of an open subset $U$ in $X$ containing $K$ such that $\mu_{y}(U)<\mu_{y}(K)+\varepsilon / 2$. Using Urysohn's lemma once again we can find a function $g \in C(X)$ with values in $[0,1]$ whose restriction to $K$ is identically equal to one while it is identically equal to zero outside $U$. Define $f \in C(X)$ by $f=e^{g}$. Clearly, $\mu_{y}(K) \leq \log \Phi(f)(y) \leq \mu_{y}(U)$. As $\log \Phi(f)$ is continuous at $y$ there exists a neighbourhood $V \subset Z$ such that $\log \Phi(f)(z)-\log \Phi(f)(y)<\varepsilon / 2$ for every $z \in V$. It follows that

$$
\mu_{z}(K)=\log e^{\int_{K} d \mu_{z}} \leq \log \Phi(f)(z)<\mu_{y}(U)+\varepsilon / 2<\mu_{y}(K)+\varepsilon, \quad z \in V .
$$

Thus, the mapping $y \mapsto \mu_{y}$ is upper semicontinuous. In almost the same way one can prove it is also lower semicontinuous.

To prove the continuity of $y \mapsto \mathcal{F}_{y}$ we choose $y \in Z$, an open and closed subset $A \subset S_{y}$ and disjoint open subsets $U, V \subset X$ such that $A \subset U$ and $S_{y} \backslash A \subset V$. We will also assume that $A \in \mathcal{F}_{y}$ since in the case that $A \notin \mathcal{F}_{y}$ the proof goes through similarly. We can find continuous functions $g, h: X \mapsto[0,1]$ such that $g_{\mid A} \equiv 1$, $g_{\mid U^{C}} \equiv 0, h_{\mid S_{y} \backslash A} \equiv 1$, and $h_{\mid V^{C}} \equiv 0$. Set $f=h-g$. Clearly, $\Phi(f)(y)=-1$. We already know that the mapping $t \mapsto S_{t}, t \in Z$, is continuous, and therefore, there exists a neighbourhood $W \subset Z$ of $y$ such that $S_{z} \subset U \cup V$ and $\Phi(f)(z)<-1 / 2$ for every $z \in W$. It follows that $\sigma_{\mathcal{F}_{z}}(f)=-1$, or equivalently, $S_{z} \cap U \in \mathcal{F}_{z}$ for every $z \in W$. Hence, $y \mapsto \mathcal{F}_{y}$ is continuous.

To prove the converse statement assume that a mapping $\Phi$ is given by (4) and that the sets $Y_{0}, Y_{1}, Z$, and the mappings $y \mapsto \mu_{y}, y \mapsto S_{y}, y \mapsto \mathcal{F}_{y}$ fulfill all the requirements of Theorem 3. In order to see that $\Phi$ is well defined we have to show that the restriction of $\Phi(f)$ to $Z$ is a continuous function for every $f \in C(X)$. So, choose $\mathbb{O}_{X} \neq f \in C(X)$ and $y \in Z$. By $Z_{f}$ we will denote the set of all zeroes of $f$. Set $M=\max _{x \in X} \log |f(x)|$.

The first possibility we will consider is that $\Phi(f)(y)=0$, or equivalently, $p=$ $\mu_{y}\left(Z_{f} \cap S_{y}\right)>0$. For every positive number $\eta<1$ we can find a neighbourhood $U$ of $Z_{f} \cap S_{y}$ such that $|f(y)|<\eta$ for every $x \in U$. Now, $\mu_{y}(U) \geq p$. Therefore, the continuity of the mapping $t \mapsto \mu_{t}$ yields the existence of a neighbourhood $V \subset Z$ of $y$ such that $\mu_{z}(X)<2 \mu_{y}(X)$ and $\mu_{z}(U)>p / 2$ for every $z \in V$. Hence, for $z \in V$ such that $f$ has no zeroes in $S_{z}$ we have

$$
\begin{aligned}
|\Phi(f)(z)| & =e^{\int_{U} \log |f(x)| d \mu_{z}} e^{\int_{X \backslash U} \log |f(x)| d \mu_{z}} \\
& \leq e^{(\log \eta) \mu_{z}(U)} e^{M \int_{X} d \mu_{z}} \leq \eta^{p / 2} e^{2 M \mu_{y}(X)} .
\end{aligned}
$$

This shows that $\Phi(f)$ is continuous at $y$.

The next possibility is that $f$ is positive on $S_{y}$. Let $\varepsilon$ be any positive number. There exists a neighbourhood $U_{0}$ of $S_{y}$ such that $1 / 2 \min _{x \in S_{y}} f(x)<f(u)<$ $2 \max _{x \in S_{y}} f(x)$ for every $u \in U_{0}$. The continuity of the mapping $t \mapsto S_{t}$ implies the existence of a neighbourhood $W \subset Z$ of $y$ such that $S_{z} \subset U_{0}$ for every $z \in W$. Set $K=\max \left\{2 \mu_{y}(X), 1+\max _{x \in S_{y}}|\log f(x)|\right\}$ and choose a positive number $\delta<7 K \mu_{y}(X)$ such that $|s-\log \Phi(f)(y)|<\delta$ yields $\left|e^{s}-\Phi(f)(y)\right|<\varepsilon$. As 
$S_{y}=\left\{x_{1}, x_{2}, \ldots\right\}$ is a countable set we can find a positive integer $N$ such that

$$
\sum_{k>N} \mu_{y}\left(\left\{x_{k}\right\}\right)<\frac{\delta}{7 K} .
$$

For every $j=1,2,3, \ldots, N$ we can find a neighbourhood $U_{j} \subset U_{0}$ of $x_{j}$ such that $\left|\log f(x)-\log f\left(x_{j}\right)\right|<\delta /(7 K)$ for every $x \in U_{j}$. Moreover, we can assume that these neighbourhoods are disjoint. As $X$ is normal and $\mu_{y}$ is regular we can also assume that $\mu_{y}\left(\bar{U}_{j}\right)<\mu_{y}\left(\left\{x_{j}\right\}\right)+2^{-j} \delta /(14 K)$. Because of the upper and lower semicontinuity of the mapping $t \mapsto \mu_{t}$ we can find for every $j=1,2,3, \ldots, N$ a neighbourhood $V_{j}$ of $y$ such that

$$
\mu_{y}\left(U_{j}\right)-2^{-j} \frac{\delta}{7 K}<\mu_{z}\left(U_{j}\right) \leq \mu_{z}\left(\bar{U}_{j}\right)<\mu_{y}\left(\bar{U}_{j}\right)+2^{-j} \frac{\delta}{14 K}<\mu_{y}\left(U_{j}\right)+2^{-j} \frac{\delta}{7 K}
$$

holds true for every $z \in V_{j}$. Finally, let $V_{0} \subset Z$ be a neighbourhood of $y$ such that $\left|\mu_{z}(X)-\mu_{y}(X)\right|<\delta /(7 K)$ for every $z \in V_{0}$. Denote $W \cap V_{0} \cap V_{1} \cap \cdots \cap V_{N}$ by $V$ and $U=\bigcup_{j=1}^{N} U_{j}$. For $z \in V$ we have $\mu_{z}(X)<\mu_{y}(X)+\delta /(7 K)<2 \mu_{y}(X) \leq K$ and

$$
\begin{aligned}
\mu_{z}(X \backslash U) & <\mu_{y}(X)+\delta /(7 K)-\sum_{j \leq N} \mu_{z}\left(U_{j}\right) \\
& <\mu_{y}(X)-\mu_{y}(U)+2 \delta /(7 K)<3 \delta /(7 K) .
\end{aligned}
$$

Hence, for every $z \in V$ we get

$$
\begin{aligned}
|\log \Phi(f)(z)-\log \Phi(f)(y)|=\left|\int_{X} \log f(x) d \mu_{z}-\int_{X} \log f(x) d \mu_{y}\right| \\
\leq\left|\int_{X \backslash U} \log f(x) d \mu_{z}\right|+\left|\int_{U} \log f(x) d \mu_{z}-\sum_{j \leq N} \log f\left(x_{j}\right) \int_{U_{j}} d \mu_{z}\right| \\
\quad+\sum_{j \leq N}\left|\log f\left(x_{j}\right)\right|\left|\mu_{z}\left(U_{j}\right)-\mu_{y}\left(U_{j}\right)\right| \\
\quad+\left|\sum_{j \leq N} \log f\left(x_{j}\right) \int_{U_{j}} d \mu_{y}-\int_{U} \log f(x) d \mu_{y}\right|+\left|\int_{X \backslash U} \log f(x) d \mu_{y}\right| \\
<\max _{x \in S_{z} \backslash U}|\log f(x)| \mu_{z}(X \backslash U)+\sum_{j \leq N} \mu_{z}\left(U_{j}\right) \delta /(7 K)+K \delta /(7 K) \sum_{j \leq N} 2^{-j} \\
\quad+\mu_{y}(U) \delta /(7 K)+\max _{x \in S_{y} \backslash U}|\log f(x)| \mu_{y}\left(S_{y} \backslash U\right)<\delta .
\end{aligned}
$$

Let us now turn to the more general case that $f$ is without zeroes on $S_{y}$. As $|\Phi(f)|=\Phi(|f|)$ the previous step shows that $|\Phi(f)|$ is a continuous function at $y$. To prove that $\Phi(f)$ is also continuous at $y$ we will find a neighbourhood $V$ of $y$ such that the mapping $z \mapsto \sigma_{\mathcal{F}_{z}}(f)$ is constant on $V$. The set $N_{S_{y}}(f)$ is open and closed in $S_{y}$. Hence, disjoint open subsets $W_{1}$ and $W_{2}$ can be found such that $N_{S_{y}}(f) \subset W_{1} \subset\{x \in X: f(x)<0\}$ and $S_{y} \backslash N_{S_{y}}(f) \subset W_{2} \subset\{x \in X: f(x)>0\}$. Let $V_{1} \subset Z$ be a neighbourhood of $y$ such that $S_{z} \subset W_{1} \cup W_{2}$ for every $z \in V_{1}$. The continuity of the mapping $t \mapsto \mathcal{F}_{t}$ guarantees the existence of a neighbourhood $V \subset V_{1}$ of $y$ such that $\sigma_{\mathcal{F}_{z}}(f)=\sigma_{\mathcal{F}_{y}}(f)$ for every $z \in V$. This completes the proof of the continuity of $\Phi(f)$ for every $f \in C(X)$. 
Once we know that $\Phi$ is well defined it is easy to see that it is multiplicative. It remains to prove that $\Phi$ is continuous. The compactness of the set $Z$ implies that it is enough to show that for every $f \in C(X)$ and every $\varepsilon>0$ and every $y \in Z$ we can find a neighbourhood $V \subset Z$ of $y$ and $\delta>0$ such that $|\Phi(f)(z)-\Phi(g)(z)|<\varepsilon$ for every $z \in V$ and any $g \in C(X)$ satisfying $\|f-g\|<\delta$. So, let us assume that $f \in C(X), y \in Z$, and $\varepsilon>0$.

The first case we will consider is that $\Phi(f)(y) \neq 0$. Using the continuity of $|f|$ and $t \mapsto S_{t}$ once again we can find a positive real number $a$ and a neighbourhood $V \subset Z$ of $y$ such that $\inf \left\{|f(x)|: x \in S_{z}\right.$ and $\left.z \in V\right\} \geq 2 a$. It follows that for $g \in C(X)$ satisfying $\|g-f\|<a$ we have $N_{S_{z}}(g)=N_{S_{z}}(f)$ for every $z \in V$. Hence,

$$
|\Phi(g)(z)-\Phi(f)(z)|=\mid e^{\int_{X} \log |g(x)| d \mu_{z}}-e^{\int_{X} \log |f(x)| d \mu_{z} \mid}
$$

for such functions $g \in C(X)$. It is easy to verify that $\mu_{t}(X) \leq\left\|\log \Phi\left(e \mathbb{1}_{X}\right)\right\|$ for every $t \in Z$. Applying this fact in (5) one can now easily conclude that for all functions $g \in C(X)$ that are sufficiently close to $f$ the inequality $\mid \Phi(g)(z)-$ $\Phi(f)(z) \mid<\varepsilon$ holds true for every $z \in V$.

To finish the proof it remains to consider the case that $\Phi(f)(y)=0$. The continuity of $\Phi(f)$ at $y$ implies the existence of a neighbourhood $V_{1} \subset Z$ of $y$ such that $|\Phi(f)(z)|<\varepsilon / 2$ for every $z \in V_{1}$. We also know that in this case $\mu_{y}\left(Z_{f} \cap S_{y}\right)=p>0$. Take any $0<\eta<1 / 2$ and let $U$ be a neighbourhood of $Z_{f}$ such that $|f(x)|<\eta$ for every $x \in U$. The mapping $t \mapsto \mu_{t}$ is continuous. Hence, one can find a neighbourhood $V \subset V_{1}$ of $y$ such that $\mu_{z}(U)>p / 2$ for every $z \in V$. Let $g \in C(X)$ satisfy $\|g-f\|<\eta$. This yields $|g(x)| \leq\|g-f\|+|f(x)|<2 \eta$ for every $x \in U$. Hence, for such $g \in C(X)$ and any $z \in V$ we have either $\Phi(g)(z)=0$ or

$$
\begin{aligned}
|\Phi(g)(z)| & =e^{\int_{U} \log |g(x)| d \mu_{z}} e^{\int_{X \backslash U} \log |g(x)| d \mu_{z}} \\
& <e^{\log (2 \eta) \mu_{z}(U)} e^{\int_{X} \log (\|g-f\|+|f(x)|) d \mu_{z}}<(2 \eta)^{p / 2} e^{\log (\eta+\|f\|)\left\|\log \Phi\left(e \mathbb{1}_{X}\right)\right\|} .
\end{aligned}
$$

Thus, for $\eta$ small enough it follows that $|\Phi(g)(z)-\Phi(f)(z)|<\varepsilon$ for every $z \in V$.

\section{REFERENCES}

1. D. G. Bourgin, Multiplicative transformations, Proc. Nat. Acad. Sci. U.S.A. 36 (1950), 564570. MR 12:421b

2. H. Goldmann and P. Šemrl, Multiplicative derivations on $C(X)$, Monatsh. Math. 121 (1996), 189-198. MR 96m:46041

3. A. N. Milgram, Multiplicative semigroups of continuous functions, Duke Math. J. 16 (1949), 377-383. MR 10:612a

4. P. Šemrl, The functional equation of multiplicative derivation is superstable on standard Banach algebras, Integr. Equat. Oper. Th. 18 (1994), 118-122. MR 94k:47055

5. P. Šemrl, Isomorphisms of standard operator algebras, Proc. Amer. Math. Soc. 123 (1995), 1851-1855. MR 95g:47066

6. A. Turowicz, Sur les fonctionelles continues et multiplicatives, Ann. Soc. Polon. Math. 20 (1947), 135-156.

Faculty of Electrical Engineering and Computer Science, University of Maribor, Smetanova ul. 17, 2000 Maribor, P. O. Box 218, Slovenia

E-mail address: gorazd.lesnjak@uni-mb.si

Faculty of Mechanical Engineering, University of Maribor, Smetanova ul. 17, 2000 Maribor, P. O. Box 224, Slovenia

E-mail address: peter.semrl@uni-mb.si 